\title{
DYNAMIC QUEUING TRANSMISSION MODEL FOR DYNAMIC NETWORK LOADING
}

\author{
Nevena Raovic ${ }^{1}$, Otto Anker Nielsen ${ }^{2}$, Carlo Giacomo Prato ${ }^{3}$ \\ ${ }^{1,2}$ Dept of Transport, Technical University of Denmark, Kongens Lyngby, Denmark \\ ${ }^{3}$ School of Civil Engineering, University of Queensland, Brisbane, Australia
}

Submitted 16 January 2015; resubmitted 13 April 2015; accepted 18 May 2015;

first published online 13 July 2015

\begin{abstract}
This paper presents a new macroscopic multi-class dynamic network loading model called Dynamic Queuing Transmission Model (DQTM). The model utilizes 'good' properties of the Dynamic Queuing Model (DQM) and the Link Transmission Model (LTM) by offering a DQM consistent with the kinematic wave theory and allowing for the representation of multiple vehicle classes, queue spillbacks and shock waves. The model assumes that a link is split into a moving part plus a queuing part, and $\mathrm{p}$ that traffic dynamics are given by a triangular fundamental diagram. A case-study is investigated and the DQTM is compared with single-class LTM, single-class DQM and multi-class DQM. Under the model assumptions, single-class models indicate that the LTM and the DQTM give similar results and that the shock wave property is properly included in the DQTM, while the multi-class models show substantially different travel times for two vehicle classes. Moreover, the results show that the travel time will be underestimated without considering the shock wave property.
\end{abstract}

Keywords: dynamic network loading; dynamic queuing transmission model; link transmission model; kinematic wave theory; queue spillbacks.

\section{Introduction}

In order to predict more realistically traffic flows and travel times on road networks, conventional static assignment models and Dynamic Traffic Assignment (DTA) models (for an extensive overview, see Peeta, Ziliaskopoulos 2001) have been developed with a focus on their application on large-scale networks. Dynamic Network Loading (DNL) models are one of the two components necessary to the process of solving DTA models (the other being the path reassignment), and they aim at propagating vehicles on the road network over time and producing as output the number of vehicles and the travel times on the links. The need for a realistic representation of traffic dynamics comes especially to the fore in the case of congested networks due to the phenomenon of queue spillbacks, which cannot be represented with a static traffic assignment.

The main challenges in the realistic representation of traffic dynamics on road networks are related to the balance between computation time and level of detail, and hence are dependent on the network size and the application of a particular model. The current study focuses on DNL models used in a planning context for large-scale applications, which are characterized by uncertainty about future data and do not require as precise a representation of traffic dynamics as the models used in real-time traffic management (Balijepalli et al. 2014). Therefore, the focus of the current study is on macroscopic DNL models, which give a less detailed traffic flow description than microscopic or mesoscopic models, but are still able to represent crucial phenomena in congested networks such as queue spillbacks.

Existing literature on DNL models proposes several approaches to represent vehicle propagation in macroscopic models, and Table 1 presents an overview of the characteristics of DNL models based on different approaches. The first attempt of time-dependent traffic flow representation is the Point Queue (PQ) model that is inspired by fluid queue models for dam processes proposed in the 1950s, is based on the bottleneck model (Vickrey 1969), and has been applied to represent traffic dynamics on traffic links (e.g., Drissi-Kaïtouni, HamedaBenchekroun 1992; Kuwahara, Akamatsu 1997; Han et al. 2013a). A variational inequality formulation of the PQ model has been recently formulated to allow the flow to be a distribution instead of an integrable func-

Corresponding author: Carlo Giacomo Prato

E-mail: c.prato@uq.edu.au 
tion (Han et al. 2013b) and has been applied to a realworld network (Han et al. 2013c). A later attempt of time-dependent and dynamic traffic flow representation was proposed in the work of Merchant and Nemhauser (1978) by introducing the concept of exit functions, where the outflow from a link is a function of the current number of vehicles on the link. Several models were developed under this theory (e.g., Smith 1984; Friesz et al. 1989). A significant number of models used travel time functions to describe vehicle propagation, where the travel time of vehicles entering a link is related to the current state and the number of vehicles on the link (e.g., Friesz et al. 1993; Astarita 1996; Wu et al. 1998; Carey et al. 2003; Carey, Ge 2007). Models based on the Kinematic Wave Theory (KWT) represented vehicle propagation as based on a fundamental diagram of traffic flow instead of a travel time function (Lighthill, Whitham 1955). Two major models were developed under this theory: the Cell Transmission Model (CTM) and the Link Transmission Model (LTM). The widely used CTM divides the links of the network into cells and propagates vehicles through the cells, thus requiring both time and space discretization (Daganzo 1994, 1995). This discretization problem may be overcome by considering whole links and avoiding space discretization in the LTM (Yperman 2007) by using Simplified KWT (SKWT) (Newell 1993a, 1993b, 1993c). The LTM represents links as a unit and describes traffic dynamics by proposing the concept of sending and receiving flows. Models based on KWT are typically representing a single class of vehicles, although an overview of multi-class KWT models can be found in the work of Logghe and Immers (2008), and a preliminary multi-class LTM model has been briefly illustrated (Smits et al. 2011) without however presenting extensive testing or results and without following up on the theory. Models based on the DQ theory were widely used in state-of-the-art DTA models (Chabini 2001; Bliemer 2007) and discussed in Zhang et al. (2013). According to the underlying theory, these models were defined Dynamic Queuing Models (DQM) where links are split into a moving part plus a queuing part. It is also relevant to mention some recent work on quasi-dynamic network loading models, which combine static traffic assignment with some dynamic phenomena such as queuing and spillbacks (Bliemer et al. 2012). Specifically, this model overcomes the drawback of static assignment via an approach that is consistent with the traffic flow theory instead of the travel time function, hence yielding a more accurate representation of queues and spillbacks in the static framework.

The aforementioned models are observed through their ability to represent queue spillbacks, shock waves, or multiple vehicle classes. Each of the models presents some drawbacks in the representation of these three characteristics, which were the motivation for the development of the model presented in this study. As illustrated in Table 1, neither the exit function nor the travel time function approach can capture these properties. This is mainly because these approaches may violate the First-In-First-Out (FIFO) rule and lead to a bias in travel time and queue spillback representation, although recent extensions of the travel time model allow for a better representation of behavior when traffic flow is varying over time (Carey et al. 2014). The models based on the KWT have overcome this limitation, but their main problem is the inability to represent multiple vehicle classes. The CTM model allows for representation of overtaking and has been recently extended to attempt capturing spillback, while the LTM model relies on the FIFO rule. However, the issue of representing multiple vehicle classes is still open and unresolved, although some research has been conducted in the direction of extending models based on the KWT to multiple vehicle classes. Moreover, the accuracy of the CTM highly depends on the length of the time step (i.e., the number of cells), which makes this model less attractive than the LTM. DQM are able to represent multiple vehicle classes, where overtaking is allowed within a moving part, but they are not able to describe shock waves and this is a drawback that can influence the realism of the queue representation. As for the compromise solution of the quasi-dynamic model, it appears suitable for planning purposes, although DTA models are able to describe traffic dynamics more accurately with acceptable computation times.

Table 1. Overview of DNL models

\begin{tabular}{|c|c|c|c|c|}
\hline Model(s) & $\begin{array}{c}\text { Traffic } \\
\text { dynamics }\end{array}$ & $\begin{array}{c}\text { Queue } \\
\text { spillbacks }\end{array}$ & $\begin{array}{l}\text { Shock wave } \\
\text { representation }\end{array}$ & $\begin{array}{l}\text { Multiple vehicle } \\
\text { classes }\end{array}$ \\
\hline $\begin{array}{l}\text { Merchant, Nemhauser (1978); } \\
\text { Smith (1984); } \\
\text { Friesz et al. (1989) }\end{array}$ & Exit function & No & No & No \\
\hline $\begin{array}{l}\text { Astarita (1996); } \\
\text { Friesz et al. (1993); } \\
\text { Wu et al. (1998) }\end{array}$ & Travel time function & No & No & No \\
\hline Point Queue Model & Vickrey's model & Yes & No & No \\
\hline Cell Transmission Model & KWT & Yes & Yes & No \\
\hline Link Transmission Model & KWT & Yes & Yes & No \\
\hline Dynamic Queuing Models & DQ (moving + queing) & Yes & No & Yes \\
\hline Dynamic Queuing Transmission Model & KWT, DQ & Yes & Yes & Yes \\
\hline
\end{tabular}


This study proposes the development of a DNL model that is able to represent shock waves and multiple vehicle classes at the same time. The developed model is called Dynamic Queuing Transmission Model (DQTM) as it combines the 'good' properties of the LTM and the DQM (Bliemer 2005). The advantage of the combination of these two models lies in the inclusion of both the shock wave property of the LTM (that the DQM does not have) and the multiple vehicle class property of the DQM (that the LTM does not have). The DQM framework allows representing multiple vehicle classes by splitting each link into a moving and a queuing part. Flows are consistent with the SKWT and each vehicle class is characterized by its own fundamental diagram. The inflow capacity changes based on the SKWT, and the queue density is calculated dynamically from the fundamental diagram on the basis of the outflow rates and is used then to calculate the length of the queuing part.

This paper is organized as follows. The next section describes the model and provides a theoretical comparison of the DQTM with the LTM and the DQM. The third section presents a case-study of a simple road network consisting of two routes, where both a single-class and a multi-class model are observed. Based on the vehicle class features, the single-class DQTM is compared with the single-class LTM and a single-class DQM, while the multi-class DQTM is compared with the multi-class DQM. The fourth section reports on the results of the case-study and highlights the differences in the representation of spillbacks and shock waves. Finally, the fifth section provides conclusions and directions for further research.

\section{Model Description}

This section focuses on the theoretical description of the proposed DQTM. Initially, a list of the notations and the definitions used in the model is provided, followed by a general description of the link and the node models.

\subsection{Notations and Definitions}

Sets:

$A$ - set of links;

$M$ - set of vehicle classes;

$N$ - set of nodes;

$P$ - set of all routes on the network;

$R$ - set of origin nodes;

$S$ - set of destination nodes;

$T$ - total simulation period.

Indices:

$a$ - link index, $a \in A$;

$m$ - vehicle class index, $m \in M$;

$n$ - node index, $n \in N$;

$p$ - route index, $p \in P$;

$r$ - origin node index, $r \in R$;

$s$ - destination node index, $s \in S$;

$i$ - time step index.
Input characteristics:

$C_{a} \quad$ - capacity of link $a[\mathrm{pcu} / \mathrm{h}]$

$k_{a}^{\text {jam }} \quad-$ jam density of link $a[\mathrm{pcu} / \mathrm{km}]$

$k_{a}^{\text {critical }}$ - critical density of link $a[\mathrm{pcu} / \mathrm{km}]$;

$L_{a} \quad$ - length of link $a[\mathrm{~km}]$;

$l_{a} \quad-$ the number of lanes on link $a$;

$q_{a}^{c} \quad$ - critical capacity of link $a[\mathrm{pcu} / \mathrm{h}]$;

$V_{a m} \quad$ - speed of vehicle class $m$ on link $a[\mathrm{~km} / \mathrm{h}]$;

$w_{a} \quad$ - backward wave speed of link $a[\mathrm{~km} / \mathrm{h}]$;

$\rho_{m} \quad$ - passenger car unit [pcu] for vehicle class $m$.

Variables:

$f_{a}(i)$ - outflow rate from link $a[\mathrm{pcu} / \mathrm{h}]$;

$f_{a m p}^{r s}(i)$ - outflow rate of class $m$ out of link $a$ at time step $i$ using route $p$ from origin $r$ to destination $s[\mathrm{veh} / \mathrm{h}] ;$

$f_{a m}(i)$ - outflow rate of class $m$ out of link $a$ at time step $i[\mathrm{veh} / \mathrm{h}]$;

$\bar{f}_{a m p}^{r s}(i)$ - potential outflow rate of class $m$ out of link $a$ at time step $i$ using route $p$ from origin $r$ to destination $s[\mathrm{veh} / \mathrm{h}]$;

$i_{a}(i)$ - potential inflow rate to link $a$ at time step $i$ [pcu/h];

$k_{a}^{\text {queue }}$ - queue density of link $a[\mathrm{pcu} / \mathrm{km}]$;

$L_{a}^{q}(i)$ - queue length on link $a$ at time step $i[\mathrm{~km}]$;

$q_{a m p}^{r s}(i)$ - inflow rate of class $m$ vehicles into the queue of link $a$ taking route $p$ from origin $r$ to destination $s$ at time step $i[\mathrm{veh} / \mathrm{h}]$;

$X_{a m}^{q}(i)$ - the number of vehicles class $m$ in the queuing part of link $a$ at time step $i$;

$X_{a}^{q}(i)$ - the number of vehicles in the queuing part of link $a$ at time step $i$;

$U_{a}(i)$ - cumulative inflow into link $a$ at time step $i$;

$F_{a}(i)$ - cumulative outflow for link $a$ at time step $i$.

\subsection{General Description}

The proposed DQTM aims at utilizing 'good' properties of the DQM and the LTM. Thus, the framework of the proposed model is inspired to the DQM framework, but with important changes that allow capturing shock waves.

Based on the available literature, the DQM embedded in the Indy software (Bliemer 2005) is used in this study in both the theoretical development of the DQTM and the case-study. The general framework of the DQM Indy considers a link as being divided into a queuing and a moving part. The lengths of these two parts are variable, and thus are calculated by simulation. The moving part is characterized by the free flow conditions, where each vehicle can travel with its given free flow speed and hence overtaking is not observed between vehicles in the same class (i.e., having the same free flow speed), while overtaking is allowed between vehicles of different classes. The queuing part is characterized by all vehicles having the same speed and the FIFO rule applies between vehicle classes. The length of the queuing part depends on the queue length, which is computed by the queue density and the number of vehicles in the queue. An important assumption of the DQM Indy is that the queue density has a fixed value and is given as 
an input. Vehicles are expressed in passenger car unit [pcu] values in order to represent different classes as a homogeneous vehicle class.

The framework of the LTM observes whole links and simply implies calculating the cumulative flows at the upstream and the downstream ends of the link. What happens in the link is therefore irrelevant, and the actual outflow can be simply obtained from the sending and the receiving flows. The flow dynamics are given by Newell's SKWT and the triangular fundamental diagram. The additional property of the LTM is its consistency with the FIFO rule, which implies ability of representing only a single vehicle class.

By combining the 'good' properties of these models, the DQTM is developed as follows. Consider a road network $G=(N, A)$ consisting of nodes $N$ and links $A$. It is assumed that the route choice is known for the entire analysis period $[0, T]$. The DQTM consists of a link and node model where, for link $a$, the input consist of link length $L_{a}[\mathrm{~km}]$, free flow speed $V_{a m}$ of vehicle class $m$ on link $a[\mathrm{~km} / \mathrm{h}]$, capacity $C_{a}[\mathrm{pcu} / \mathrm{h}]$, jam density $k_{a}^{\text {jam }}$ $[\mathrm{pcu} / \mathrm{km}]$. The link and the node models are detailed in the following sections.

\subsection{Link Model}

The flow-density relationships on the links are captured by the triangular fundamental diagram which is given for each vehicle class $m$ and link $a$. The fundamental diagram of two vehicle classes is represented in Fig. 1. It is assumed that each link $a$ is divided into a moving and a queuing part, which are characterized by the free flow and congested regimes of the fundamental diagram, respectively. Each vehicle class $m$ is characterized by the free flow speed $V_{a m}$, which remains the same over the moving part of link $a$ to ensure the FIFO rule within the same vehicle class. On the other hand, overtaking is possible between different classes (e.g., cars can overtake trucks) as in the DQM Indy. The backward wave speed $w_{a}$ is given for each link $a$, and it is the same for all vehicle types traversing the link. This implies that the slope of the second part of the fundamental diagram representing the congested regime is the same for the fundamental diagrams of different vehicle classes on link $a$, and this further implies that there is no overtaking in the queuing part of link $a$, where the FIFO rule applies to all vehicle classes (e.g., cars stay in queue behind trucks).
In order to calculate the real inflow to the link and the real outflow from the link, it is necessary to know the queue length $L_{a}^{q}(i)$, the potential outflow $\bar{f}_{a m p}^{r s}(i)$ $[\mathrm{pcu} / \mathrm{sec}]$ given for each route $p$, origin-destination pair $(r, s)$, link $a$, and vehicle class $m$, and the potential inflow $i_{a}(i)[\mathrm{pcu} / \mathrm{sec}]$. These variables are calculated in the link model, and then used in the node model to obtain the actual inflows and outflows.

The queue length is needed in order to determine the length of the queuing part, and hence the length of the moving part, and it is expressed as:

$$
L_{a}^{q}(i)=\frac{\sum_{m} \rho_{m} X_{a m}^{q}(i-1)}{l_{a} k_{a}^{\text {queue }}(i)},
$$

where: $\rho_{m}$ is measured as [pcu] for vehicle type $m$; $X_{a m}^{q}(i-1)$ is the number of vehicles of class $m$ in the queuing part of link $a$ at time step $i-1 ; l_{a}$ represents the number of lanes on link $a$; $k_{a}^{\text {queue }}$ is the queue density on link $a[\mathrm{pcu} / \mathrm{km}]$.

Compared to DQM Indy, the queue density is not given exogenously as a fixed value, but it is calculated from the fundamental diagram and expressed in $[\mathrm{pcu}]$ as follows:

$$
k_{a}^{\text {queue }}(i)=k_{a}^{\text {critical }}+\frac{q_{a}^{c}-f_{a}(i-1)}{q_{a}^{c}}\left(k_{a}^{\text {jam }}-k_{a}^{\text {critical }}\right),
$$

where: $k_{a}^{\text {critical }}$ is the density $[\mathrm{pcu} / \mathrm{km}]$ that corresponds to the link capacity; $q_{a}^{c}$ is the capacity flow for link $a$ $[\mathrm{pcu} / \mathrm{h}] ; k_{a}^{j a m}$ is the jam density [pcu/km].

All the variables are given as input, with the exception of the outflow rate $f_{a}(i-1)$ that is calculated in the previous time step $(i-1)$.

Once the queue length is computed, the potential inflow and the potential outflow can be calculated. The potential outflow represents the flow that would leave a link if there were no capacity constraints on the following link. It is given by the expression from the DQM Indy, and it is characterized by two possible cases, namely whether there is a queue or not. The first case implies that, if there is no queue on the link, the outflow rates for the vehicle classes are equal to the inflow into the tail of the queue, where the tail of the queue is actually the downstream end of the link. The second case implies that, if there is a queue, the path-specific outflow rate for vehicle class $m$ is given by the share of the capacity

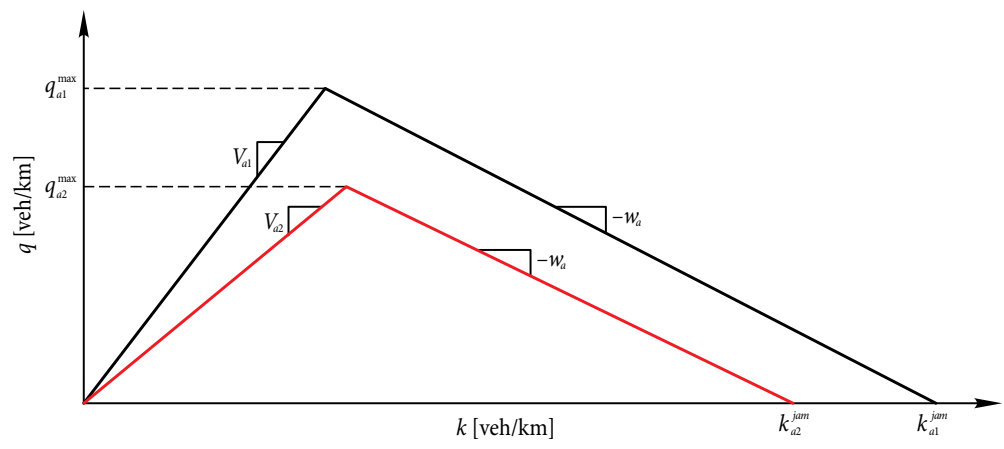

Fig. 1. The fundamental diagram for two vehicle classes 
between different vehicle classes that are on the head of the queue. The potential outflow rate can be expressed as follows:

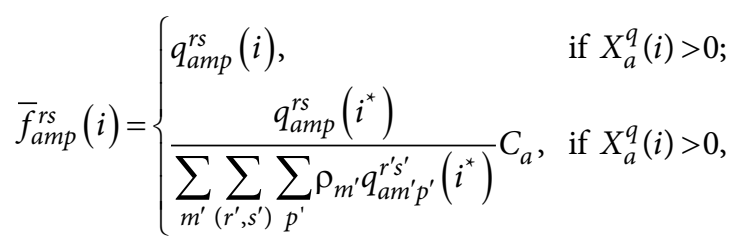

where: $X_{a}^{q}(i)$ is the total number of vehicles in the queuing part of link $a$ at time step $i$ expressed in [pcu]; $q_{a m p}^{r s}(i)$ is the path-specific inflow into the tail of the queue for vehicle class $m$ at time step $i$; $q_{a m p}^{r s}\left(i^{*}\right)$ is the path-specific inflow into the tail of the queue for vehicle class $m$ at time step $i^{*}$ which coincide with the flow that is currently (at time step $i$ ) at the head of the queue.

The potential inflow is defined using the idea of the receiving flow from the LTM. It represents the flow that would be accepted to the link if there was an infinite capacity on the link. It is based on the Newell's SKWT and says that, ' $[\ldots]$ if a congested traffic state occurs at the upstream boundary of the link at time $t+\Delta t$ it must be emitted from the downstream boundary $-\frac{L_{a}}{w_{a}}$ time units earlier, since a congested traffic state travels with a (negative) speed 'w' (Yperman 2007). Accordingly, the following expression defines the potential inflow $[\mathrm{pcu} / \mathrm{sec}$.$] as the minimum of the difference between the$ cumulative flows at the upstream and the downstream ends of the link and the capacity of link $a$ divided by time step $\Delta t$ :

$i_{a}(i)=\frac{\min \left(F_{a}\left(i+1-\frac{L_{a}^{q}(i)}{w_{a} \Delta t}\right)+k_{a}^{j a m} L_{a}^{q}(i)-U_{a}(i), q_{a}^{c} \Delta t\right)}{\Delta t}$,

where: $\Delta t$ is the time step; $F_{a}\left(i+1-\frac{L_{a}^{q}(i)}{w_{a} \Delta t}\right)$ is the cumulative outflow for link $a$ at time step $i+1-\frac{L_{a}^{q}(i)}{w_{a} \Delta t}$; $k_{a}^{\text {jam }} L_{a}^{q}(i)$ represents the maximum flow that can be on link $a$ [pcu]; $U_{a}(i)$ is the cumulative inflow into link $a$ at time step $i$. The component $q_{a}^{c} \Delta t$ refers to the number of vehicles corresponding to the capacity rate $q_{a}^{c}$ $[\mathrm{pcu} / \mathrm{sec}]$ that can be sent at time step $\Delta t$. It should be noted that the potential inflow expressed in Eq. (4) depends on the assumption that the backward wave speeds are equal across vehicles of different classes.

The outflow rate is calculated for each vehicle class $m$ and can be translated into [pcu] values using the following expression:

$$
f_{a}(i-1)=\sum_{(r, s)} \sum_{(p)} \sum_{m} \rho_{m} f_{a m p}^{r s}(i-1) .
$$

Considering that this is a very simple calculation, it will not increase the computation time substantially even for large-scale networks.

\subsection{Node Model}

In the node model, the actual outflow and inflow rates are calculated. The actual outflow depends on both the potential inflow and potential outflow. If there is no congestion, potential inflow will be higher or equal to the potential outflow of link a, and all vehicles that want to leave the link a will actually leave. In other words, the potential outflow will be the actual outflow. In the opposite case, when the potential outflow is higher than the potential inflow, the actual outflow will be constrained by the potential inflow. This means that some vehicles will stay on the link $a$, and a queue will form. In this way, the congestion will be located at the downstream end of the link and propagated with the backward wave speed $w$ towards the upstream end of the link. Based on the queue length that forms in such case (calculated with (1)), the length of $a$ queuing and $a$ moving part will be calculated. The actual inflow is simply the actual outflow from the previous links on the same route.

Although apparently similar to the DQM Indy, very important changes have been made and, as supported by the example in Section 2, they lead to a more realistic output. Firstly, the queue density is assumed to be fixed in the DQM, while in the DQTM it is calculated on the basis of the link outflow expressed in Eq. (2). Secondly, the potential inflow expressed in Eq. (5) is calculated using the Newell's SKWT, which is also used in the LTM to calculate the receiving flow, but only for the singleclass model. In the DQM, this variable is represented by the inflow capacity, depends on the queue length and simply takes the capacity value if the queue length is shorter than the link length, and zero if the queue length is higher than the link length (i.e., if there is a queue spillback). In the DQTM there is no reliance on the queue length, but rather on the shock wave effect described above. The queue length is however calculated in order to use the framework of the multi-class DQM.

\section{Case-Study}

A case-study is considered using the same network example presented in Fig. 2. Firstly, the case-study presents a comparison of three single-class models: LTM, DQM Indy and DQTM. Secondly, the case-study proposes a comparison of a multi-class DQTM and a multi-class DQM Indy, since LTM is not able to represent multiple vehicle classes. The network consists of one origin-destination pair and two routes. Route 1 includes links 1 , 2, 4 and 6, while route 2 includes links $1,3,5$ and 6 . As illustrated in Table 1, link 1 and link 6 have three lanes and the largest capacity, links 2, 3, and 5, have two lanes, while link 4 has only one lane and hence acts as a bottle-

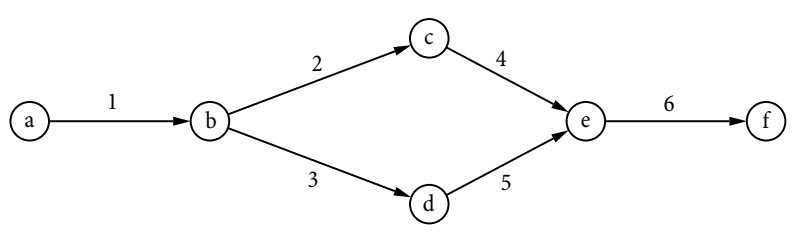

Fig. 2. Network configuration 
neck. The lane drop on the link 4 will cause congestion and potential queue spillback to the upstream links. For the single-class case-study, only the free flow speed value for class 1 from Table 1 is considered.

The demand for each route is presented in Table 2 for both the single-class and the multi-class models. The vehicles enter the network during the first 1200 seconds of the full simulation time and, in the period between 300 and 600 seconds of the simulation, the demand exceeds the capacity of link 4 . Before and after this period, the demand is less than the network capacity. The simulation is completed when all vehicles are propagated through the network.

\section{Results}

The following figures represent the results of the comparisons that focus on the travel time and the cumulative flows on the network links.

The first comparison concerns the single-class LTM, DQM Indy and DQTM, and Fig. 3 represents the travel times obtained for these three models, each route and each vehicle class. It can be seen that there is consistency in the results obtained from the LTM and the DQTM. Specifically, in both the LTM and the DQTM the queue on route 1 starts forming from about 150 seconds and the vehicles with this departure time experience longer travel time. The queue formation then follows the increase in demand which is observed from 300 seconds to 600 seconds. The queue on route 2 starts forming together with the increase in the demand. Given the network characteristics presented in Table 2 and the demand showed in Table 3 , it is expected a bottleneck to appear at link 4, and hence it is expected that travelers on route 1 will experience longer travel time. The de- mand drops after 600 seconds and the queues start dissipating. Since route 1 has a longer queue, it takes more time for its queue to resolve. The difference between the LTM and the DQTM is in the peak values of travel times for both routes. The similarity between the results in these two models can be explained by the same underlying assumptions that describe vehicle propagation. In both models, traffic dynamics are given by the triangular fundamental diagram. The additional property of the DQTM is the explicit representation of queues since it uses the DQM framework where links are divided into queuing and moving part. Compared to the LTM and the DQTM, the DQM Indy model gives significantly different results. The travel time is shorter for both routes, and the queues start forming later and dissipate faster. The queues on both routes start forming at the same time. The peak values are significantly smaller compared to the peak values in the other two models, especially for the route 2 where the longest travel time reaches 100 seconds, which is only 10 seconds more than the free flow travel time.

Figs 4-6 represent the cumulative inflows and cumulative outflows for each link obtained for the LTM, DQTM and DQM Indy, respectively. Figs 5-6 contain additional information on cumulative flows entering the queue, since there is an explicit queue representation in those two models.

Fig. 4 represents cumulative inflows and outflows for the LTM. In general, the smaller the difference between these two curves, the faster a vehicle travels over a specific link. The horizontal distance between those two curves represents the time that a vehicle spends on the link. When there is no queue, this time is equal to the free flow travel time. When the queue appears, this

Table 2. Network characteristics

\begin{tabular}{|c|c|c|c|c|c|c|}
\hline Link characteristics & Link 1 & Link 2 & Link 3 & Link 4 & Link 5 & Link 6 \\
\hline Length $[\mathrm{km}]$ & 0.9 & 0.6 & 0.6 & 0.3 & 0.3 & 0.9 \\
\hline Capacity [pcu/h] & 5400 & 3600 & 3600 & 3600 & 1800 & 5400 \\
\hline Number of lanes & 3 & 2 & 2 & 1 & 2 & 3 \\
\hline Jam density [pcu/km] & 130 & 130 & 130 & 130 & 130 & 130 \\
\hline Free flow speed class $1[\mathrm{~km} / \mathrm{h}]$ & 108 & 108 & 108 & 108 & 108 & 108 \\
\hline Free flow speed class $2[\mathrm{~km} / \mathrm{h}]$ & 54 & 54 & 54 & 54 & 54 & 54 \\
\hline Backward wave speed $[\mathrm{km} / \mathrm{h}]$ & 36 & 36 & 36 & 36 & 36 & 36 \\
\hline
\end{tabular}

Table 3. Demand characteristics

\begin{tabular}{|c|c|c|c|c|c|}
\hline \multirow{2}{*}{ Model } & \multirow{2}{*}{ Time intervals $[\mathrm{s}]$} & \multicolumn{2}{|c|}{ Demand - Route 1 [veh/h] } & \multicolumn{2}{|c|}{ Demand - Route $2[\mathrm{veh} / \mathrm{h}]$} \\
\hline & & Car & Truck & Car & Truck \\
\hline \multirow{3}{*}{ Single-class models } & $0-300$ & 1600 & - & 2200 & - \\
\hline & $300-600$ & 2300 & - & 2800 & - \\
\hline & $600-1200$ & 1000 & - & 1200 & - \\
\hline \multirow{3}{*}{ Multi-class models } & $0-300$ & 1000 & 200 & 1200 & 300 \\
\hline & $300-600$ & 1400 & 350 & 1600 & 400 \\
\hline & $600-1200$ & 800 & 100 & 1000 & 100 \\
\hline
\end{tabular}



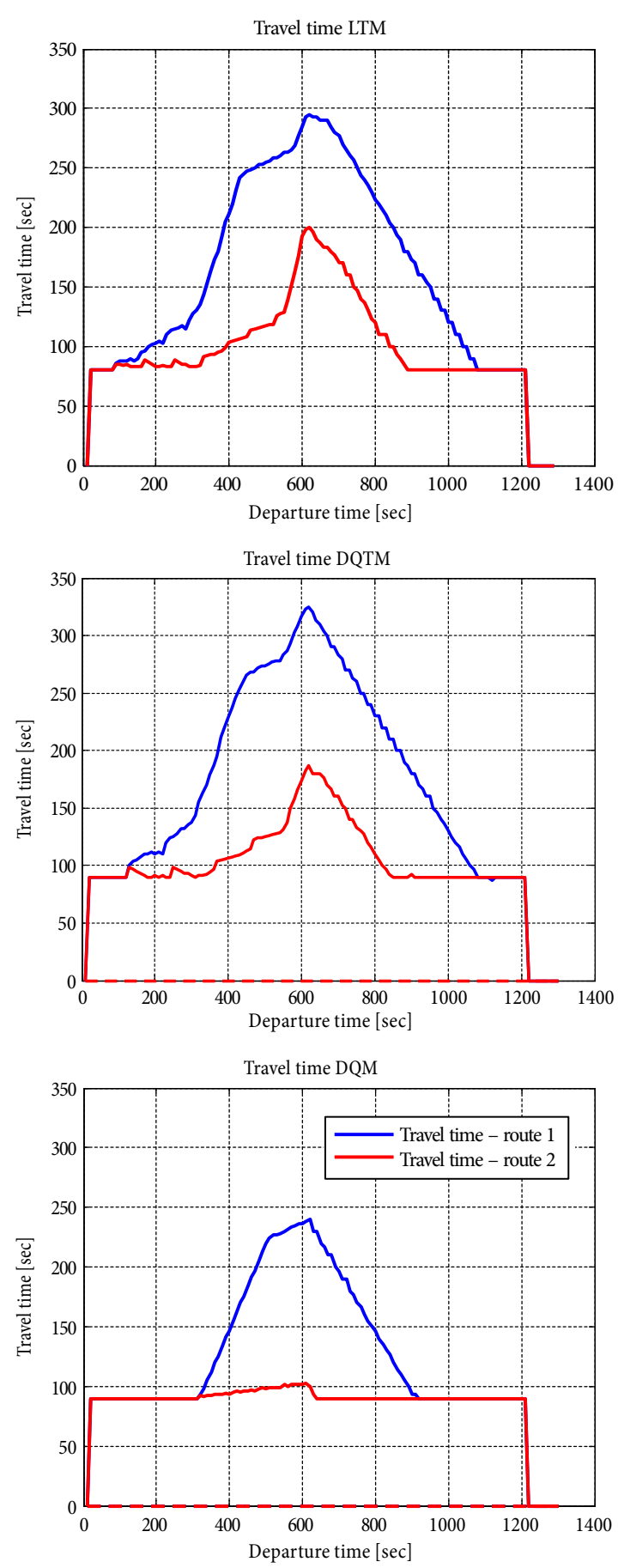

Fig. 3. Travel time for the single-class models

distance will become larger (see, e.g., link 2) and will include also a waiting time spent in the queue. It can be noticed from Fig. 4 that the significant queues form on link 1, link 2 and link 4, while link 3 and 5 have very short queues. The queues on these links appear due to reduced capacity (or potential inflow) on the following link. However, this capacity drop has different origins. The queue on link 2 forms due to the bottleneck on link 4 , and the bottleneck effect is even larger due to the queue on link 4 . The queue on link 4, however, has an origin in the shock wave effect. A shock wave influences the capacity of a specific link, and hence can cause a queue on the previous link. It is especially easy for a queue to occur on link 4 since it has only one lane. The shock wave on link 5 causes a small queue on link 3 . Link 1 has a queue from 450 to around 850 seconds, not due to the spillback from link 2 (the queue on link 2 does not reach the link length), but due to the reduced capacity and potential inflow on link 2 . These graphs also explain the travel time for LTM in Fig. 1. We can see that the queue on link 2 starts forming from 400 seconds and it is present until almost the end of the simulation. Also, link 4 has a queue from almost the beginning to the end of the simulation. This explains the much longer travel time on route 1 than on route 2 , since link 3 and link 5 have only negligible queues. Only link 6 does not have queues since it is assumed that the capacity after this last link is infinite, so all vehicles on link 6 will always travel at the free flow speed.

The results obtained for the DQTM are represented in Fig. 5 and are very similar to the LTM case. Fig. 5 contains only additional information on cumulative flows entering the queue for each link, since there is an explicit queue representation in the DQTM. When there is no queue, this curve overlaps with the cumulative link outflow curve. In this case, the vehicles enter an imaginary queue located at the end of the link, and leave it automatically. However, when there is a queue, there will be a difference between these two curves showing the travel time spent in the queue (horizontal difference) and the number of vehicles in the queue (vertical difference). When there is a queue, the cumulative link inflow curve and the cumulative queue inflow curve will be closer, indicating a reduced free flow travel time on the link. As in the LTM, the significant queues form on links 1,2 and 4 , and very small queues form on link 3 and link 5 . Again, the shock wave effect from link 6 has a higher influence on link 4 than link 5 , since it has only one lane and half the capacity of link 5 . As previously discussed, link 6 does not present any queues.

Fig. 6 represents the cumulative flow for the singleclass DQM Indy. The obtained results are significantly different compared with the results from the other two models. The first difference is that queues do not form on links 1 and 3. Moreover, the queues that form on links 2, 4 and 5 are substantially shorter. The reason behind this is that the shock wave property is not included in the DQM Indy. The queues that form are due to the lane drop on link 4 . The travelers on route 2 will also experience some waiting time on link 5 , since links 4 and 5 merge into link 6 and a queue on link 4 can also block link 5. As a result, vehicles propagate faster and have shorter waiting time and travel time. However, the absence of queues on links 1 and 3 influences the travel time on route 2 significantly, which can explain close to free flow travel time on this route (Fig. 3).

Although the LTM and the DQTM present similar results, we can observe that, the latter is able not only to capture the shock wave effect and to represent the queues explicitly, but also to account for multiple vehicle classes. Accordingly, the second comparison concerns the multi-class DQTM and the multi-class DQM Indy. 

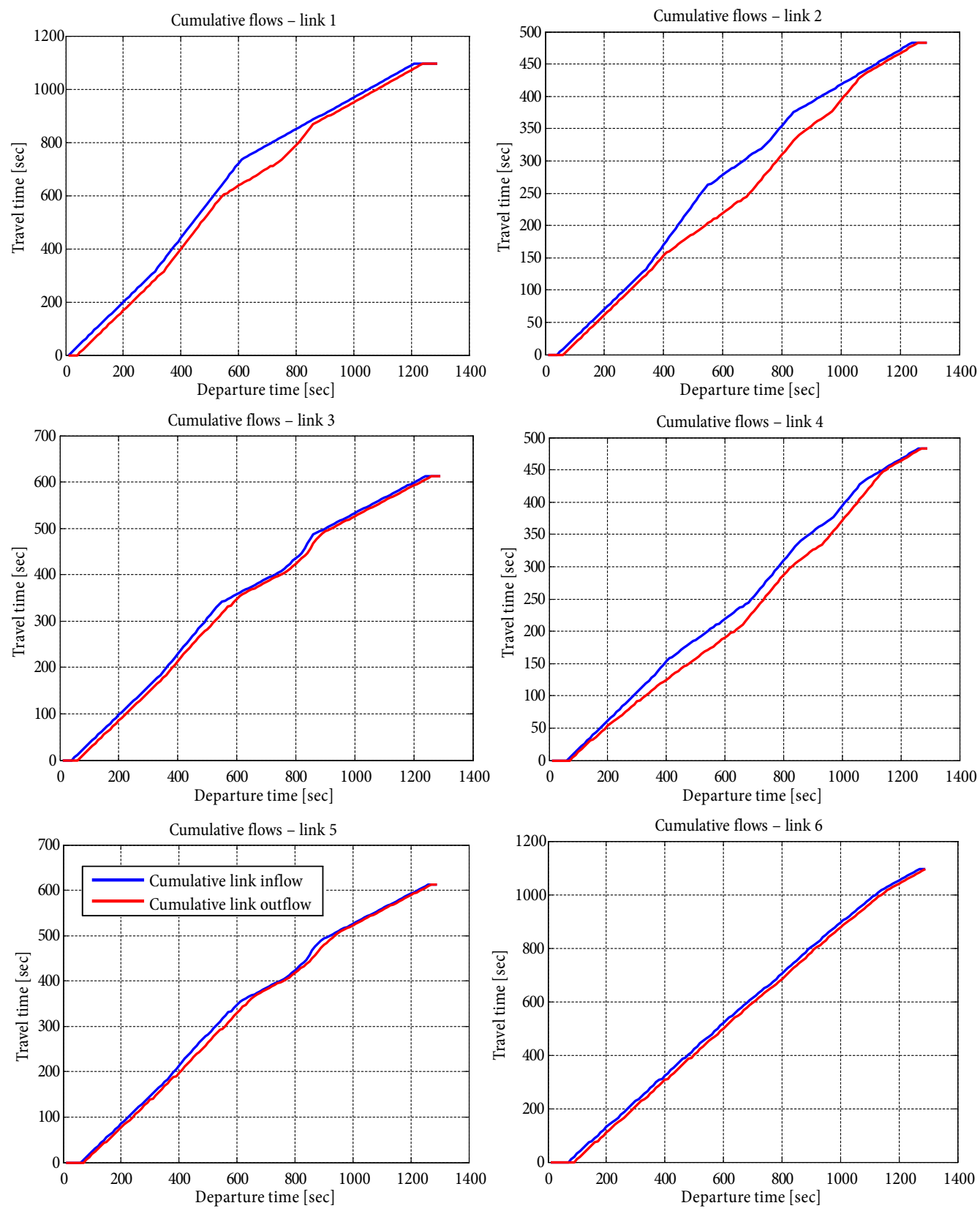

Fig. 4. Cumulative flows for single-class LTM

Fig. 7 represents the travel times obtained for each route and each vehicle type for the DQTM and the DQM. It can be noticed a significant difference in travel times between different vehicle classes, with class 2 having a higher travel time for both routes and both models. Since this vehicle class has indeed a lower speed (Table 2), the longer travel time is correctly captured by the models. However, the values of the travel times for the two models are substantially different. For route 1 , the travel time in both models has a free flow value (90 seconds for class 1 and 180 seconds for class 2) for around 300 seconds of the simulation (when the demand is less than the network capacity) and starts increasing with the increase in the demand. However, it can be seen that the DQTM has a higher travel time on route 1 than the DQM for both vehicle classes. For class 1, the travel time reaches around 320 seconds in the DQTM, while in the DQM it is around 200 seconds. For class 2, the difference is the same, only that the travel times are even higher and reach 400 seconds for the DQTM and about 325 seconds for the DQM. Moreover, the queue on route 1 dissolves more slowly in the DQTM and it is present until almost the end of the departure time (since the travel time is higher than the free flow travel time until the end of the departure time period). As for route 2, in the DQM both vehicle classes travel with their free flow speeds during the entire departure time period and there is no queue. In the DQTM, a queue forms on route 2 as well, and it results in the increased travel time for both vehicle classes. For class 1, the vehicles departing after 300 seconds experience a queue, which is present until 900 seconds of the departure time. For class 2, the 

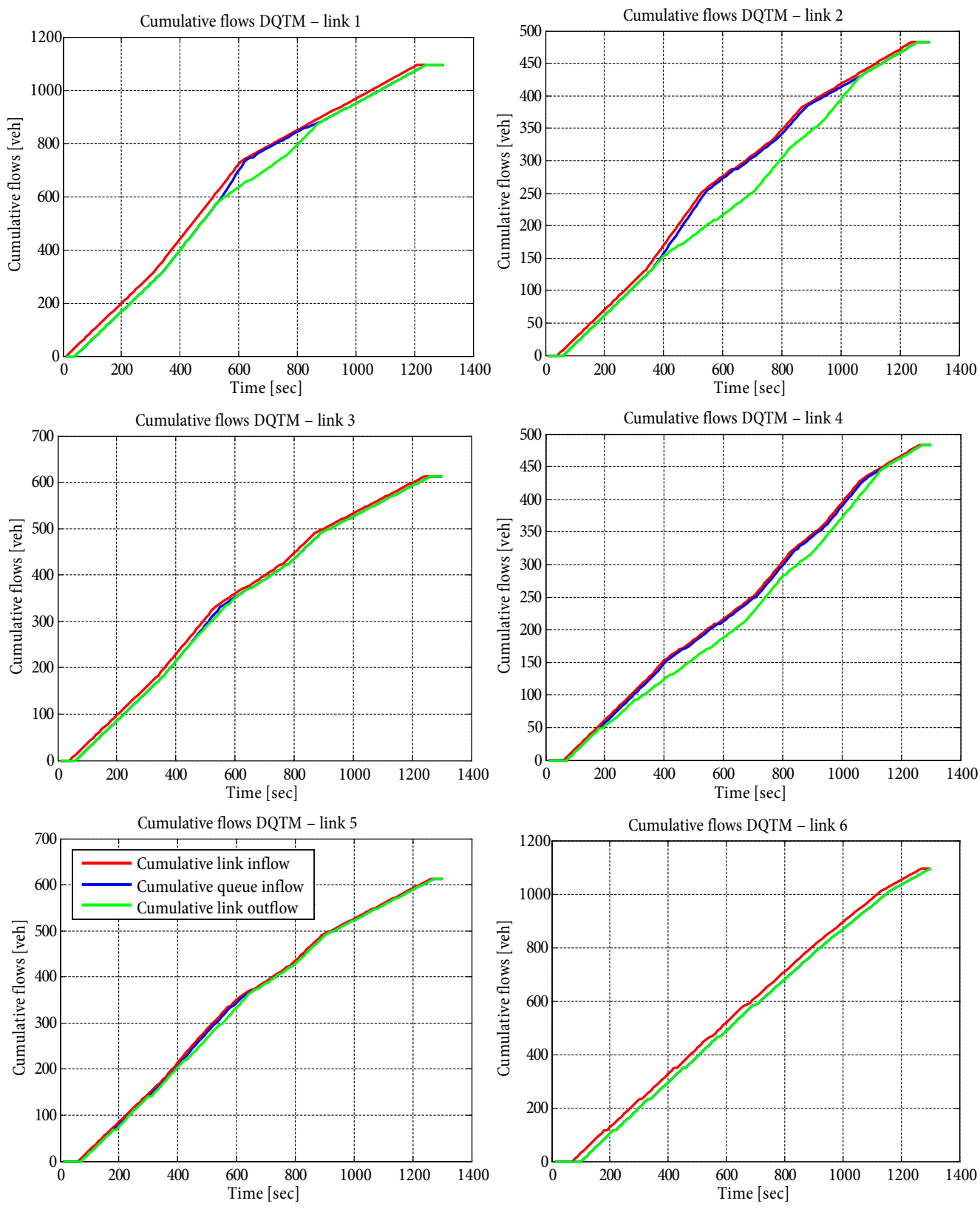

Fig. 5. Cumulative flows for single-class DQTM

queue forms and dissolves a little bit later. Noticeably, it can be concluded that the models give quite different output and the following figures explain the origin of the differences in more detail.

As in the single-class case, the multi-class models are observed thorough their cumulative link values. As both DQM Indy and DQTM can present queues explicitly, three cumulative link values are considered in Fig. 8 for the DQTM and Fig. 9 for the DQM: cumulative link inflow, cumulative queue inflow and cumulative link outflow. In the DQTM the queues form for both vehicle classes on all links with the exception of link 6 . The origins of these queues are the same as in the singleclass case. The queue on link 2 is the longest due to the presence of the lane drop on link 4, as well as the shock wave from link 2 and the reduced potential inflow to link 4. The queues on the other links are caused by the shock waves on the respective previous links. Further, the cumulative number of vehicles for class 2 are smaller than for class 1 for all links, and this is due to the lower demand of this vehicle type (Table 3). It can be noticed from Fig. 8 that the queues for both vehicle classes are present during similar time periods for all links. Also, the waiting time in the queues is almost the same for the two classes, which is indicated by the horizontal difference between the cumulative queue inflow and $\mathrm{cu}$ mulative link outflow. However, the vertical difference is significantly smaller for class 2 , indicating the smaller number of vehicles of class 2 on the network. In some cases, class 1 has even a longer waiting time. This is because this class is faster and overtakes vehicles from class 2 on the moving part of the links, and hence arrives first 

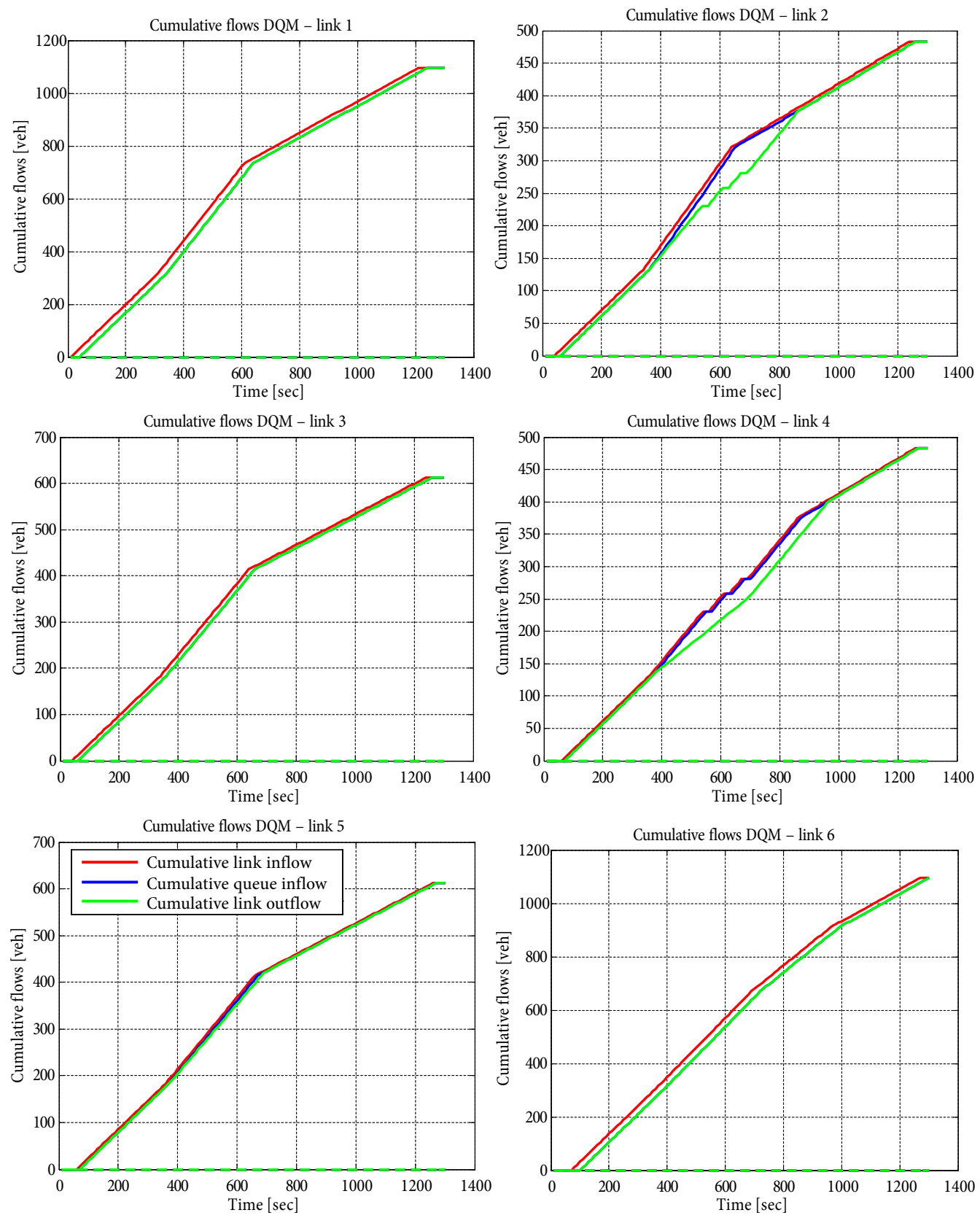

Fig. 6. Cumulative flows for single-class DQM
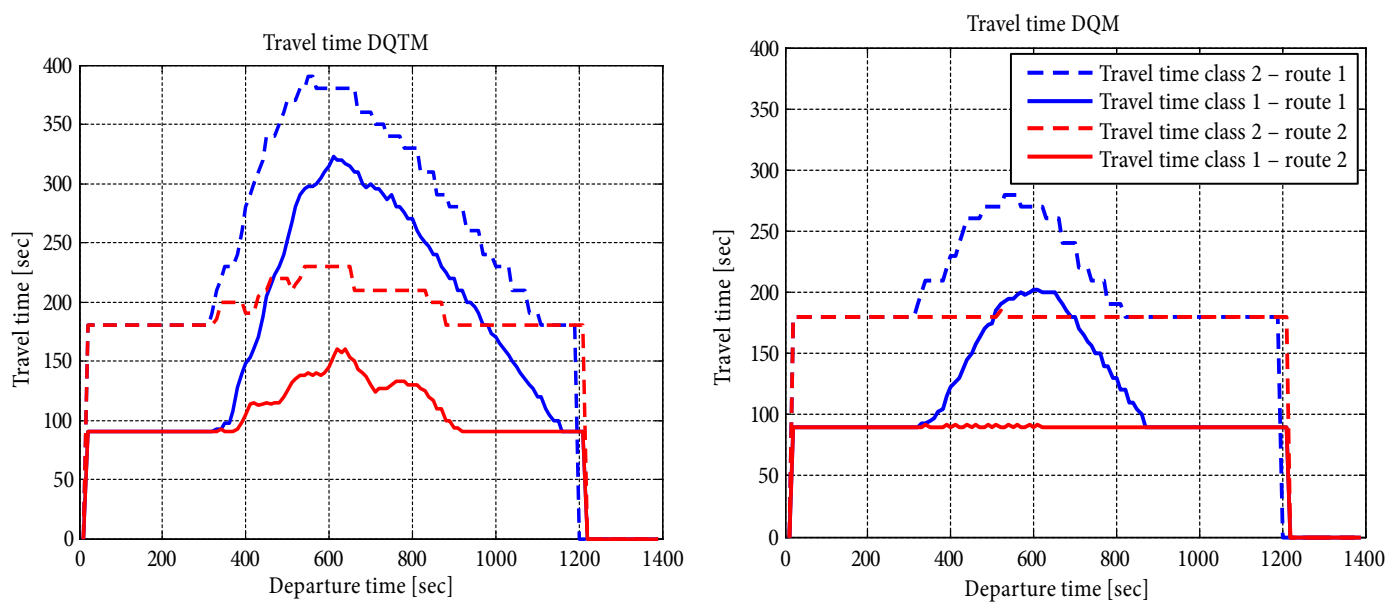

Fig. 7. Travel time for multi-class DQTM and DQM 
into the tail of the queue and then experiences longer waiting time. This phenomenon can be observed also on Fig. 6 for the DQTM, where class 1 on route 2 has a jump in the travel time from 90 seconds (free flow travel time) to 160 seconds, while class 2 has a jump from 180 seconds (free flow travel time) to 225 seconds. However, a very similar waiting time in the queue is due to the FIFO rule in the queuing part. The different moment of arrival into the tail of the queue results in longer waiting time for faster vehicle class.

The same plots are given for the multi-class DQM in Fig. 9. In this model, for the same demand, queues form only on link 2 and link 4, which explains the free flow travel time on route 2 throughout the simulation.
Even the queues that form on links 2 and 4 are smaller and present during a shorter time period compared to the queues on these links in the DQTM (Fig. 8). The significantly different results can be explained by the representation of shock waves in the DQTM. First of all, the queues on the other links that appear in the DQTM are due to this property, and hence cannot be captured by the DQM. Furthermore, the queues that exist in both models are different due to a different representation of link capacities in these models and again, the shock waves influence the value of the potential inflow in the DQTM. Queues dissipate slowlier in the DQTM due to the fact that empty car slots are not automatically noticed by the vehicle behind it, but this information is
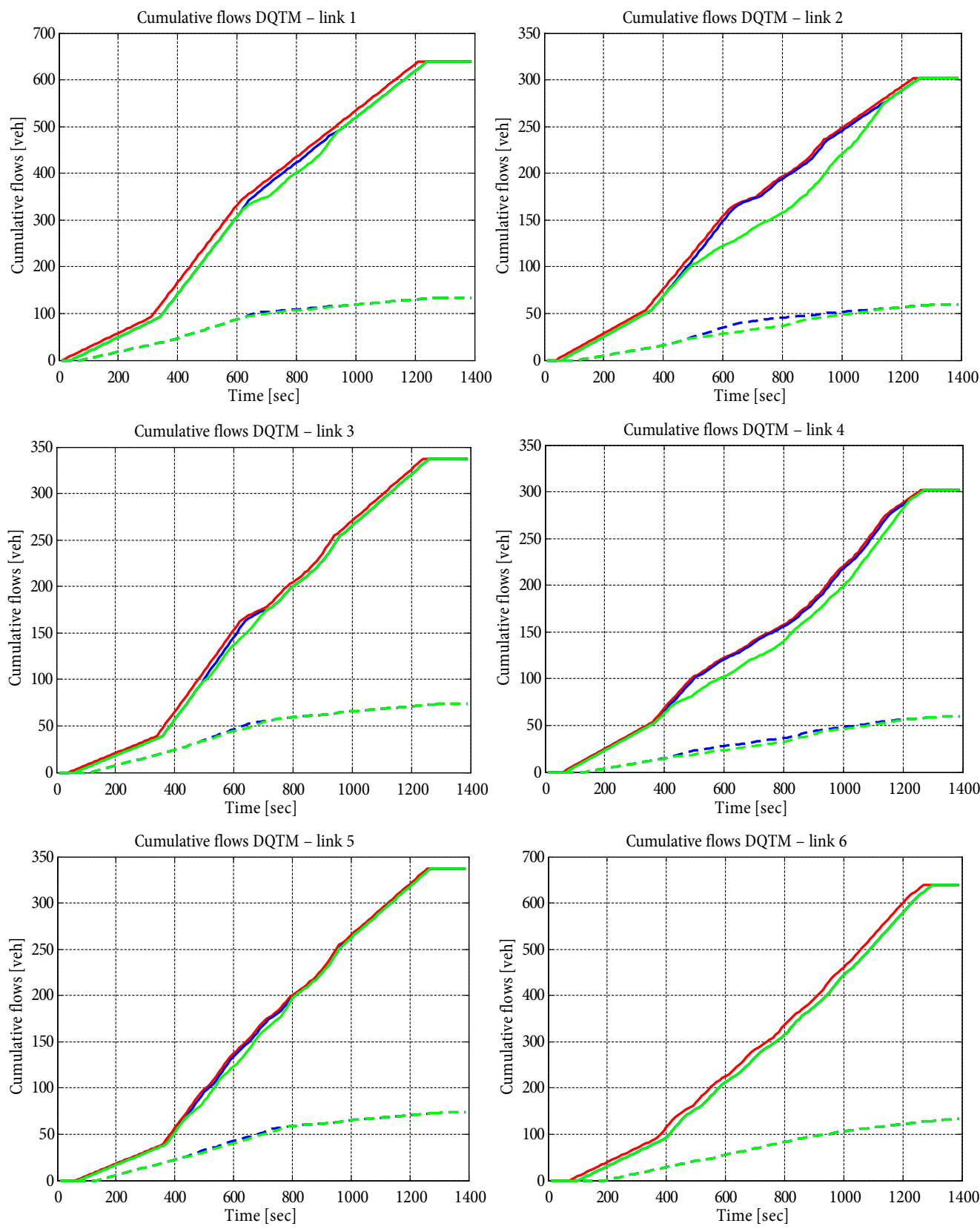

Cumulative inflow - class 1
- - Cumulative inflow - class 2

Cumulative queue inflow - class 1
- - Cumulative queue inflow - class 2

Cumulative outflow - class 1

- - Cumulative outflow - class 2

Fig. 8. Cumulative flows for multi-class DQTM 
propagated with the backward wave speed. On the other hand, in the DQM an empty car slot is automatically noticed, even if a queue is very long. This is counterintuitive, since it takes some time for all the vehicles in a queue to advance and make space for a new vehicle at the tail of the queue. This indicates that queues dissipate in different directions in the DQTM and the DQM, as also noticed for the LTM an the DQM Indy by Yperman (2007). The queues in the DQM Indy dissipate from the tail, while the queues in the DQTM dissipate from the head of the queue.

\section{Summary and Conclusions}

The realistic vehicles propagation in a congested network plays an important role in transportation network analysis. During the last few decades, research has been conducted in order to represent traffic dynamics, queues and spillbacks as realistically as possible. Macroscopic DNL models offer very well-known CTM and LTM as the models able to capture the shock wave effect, but not able to take multiple vehicle classes into account. On the other hand, DQM suggest a different approach on
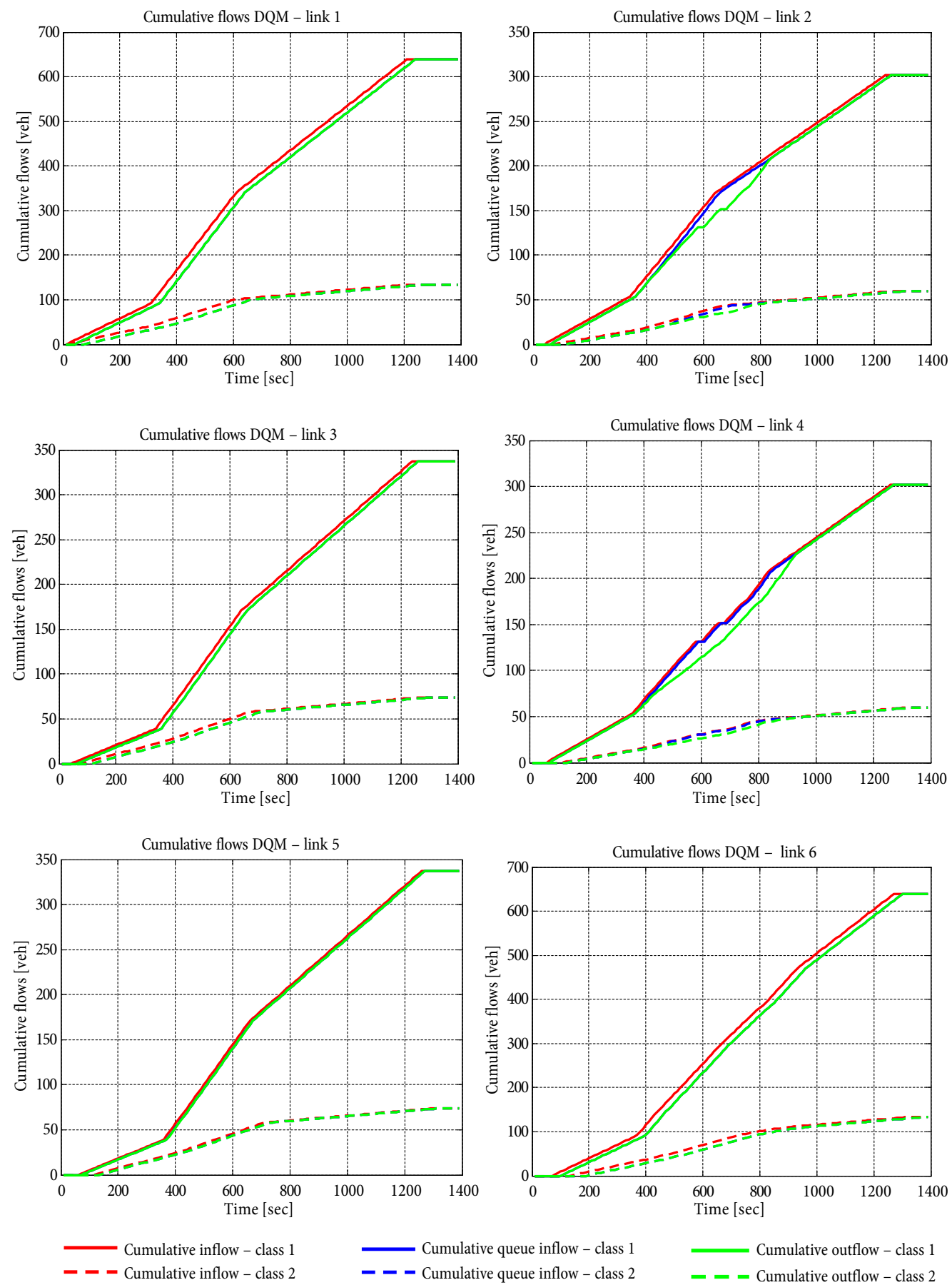

Fig. 9. Cumulative flows for multi-class DQM 
vehicle propagation without capturing shock waves, but being able to include multiple vehicle types.

This paper offers a new DNL model called DQTM. The main advantage of the proposed model lies in its ability to represent both multiple vehicle classes and shock waves, as the existing models deal with only either one of these properties. The model has been applied to a simple network problem where it is compared with the LTM and the DQM from the INDY software package through a case-study.

The first comparison deals with the single-class LTM, DQM and DQTM, while the second case-study compares multi-class DQTM and multi-class DQM. The first case-study indicates that the LTM and the DQTM give similar results and hence that the shock wave property is properly included in the DQTM. Substantially different results obtained by the single-class DQM show the importance of capturing shock waves, and that the travel time will be underestimated without this property.

The second comparison indicates that the DQTM is able to represent multiple vehicle types. Namely, the significant difference in the travel times for different vehicle types in both models indicates the importance of the multi-class DNL model. This difference appears due to different free flow travel times, and also different moment of vehicle arrival into the tail of the queue. A faster vehicle can experience longer waiting time, because it can overtake a slower vehicle type on a moving part and enter a queuing part earlier. However, the travel times obtained by the DQTM and the DQM are, as expected, very different. The shock wave effect in DQTM plays a tremendous role in the vehicle propagation. It ensures a more realistic representation of queue formation and dissipation and gives more accurate travel time as an output. The DQM Indy gives shorter travel time compared to both the single-class LTM and DQTM, and the multi-class DQTM. This shows that the absence of shock wave property in the model can underestimate travel time.

Lastly, the computation performances of the models are comparable on the small-scale test network, and further research is needed to implement the model in large-scale networks. Moreover, depending on the model application, there are several aspects that can be further developed for the application of the model. If the model is applied in practice, the triangular fundamental diagram can be modified based on the empirical data, so that it can more accurately describe a specific flow-density relationship. In addition, an explicit representation of lane groups would lead to a more realistic output. Namely, it could happen that a queue in one turning movement artificially blocks vehicles in other movements if there is no explicit queue representation throughout different lane groups. Finally, as the KWT is usually used to describe traffic dynamics on motorway networks, the model can be extended in order to include intersection modelling, and hence can be applied also to urban networks.

\section{References}

Astarita, V. 1996. A continuous time link model for dynamic network loading based on travel time function, in Proceedings of the 13th International Symposium on Transportation and Traffic Theory, 24-26 July 1996, Lyon, France, 79-101.

Balijepalli, N. C.; Ngoduy, D.; Watling, D. P. 2014. The two-regime transmission model for network loading in dynamic traffic assignment problems, Transportmetrica A: Transport Science 10(7): 563-584.

http://dx.doi.org/10.1080/18128602.2012.751680

Bliemer, M. 2007. Dynamic queuing and spillback in analytical multiclass dynamic network loading model, Transportation Research Record 2029: 14-21. http://dx.doi.org/10.3141/2029-02

Bliemer, M. C. J. 2005. INDY 2.0 Model Specifications. Working Report, Delft University of Technology, The Netherlands.

Bliemer, M.; Brederode, L.; Wismans, L.; Smits, E.-S. 2012. Quasi-dynamic network loading: adding queuing and spillback to static traffic assignment, in TRB 91st Annual Meeting Compendium of Papers DVD, 22-26 January 2012, Washington, DC, 1-24.

Carey, M.; Ge, Y. E. 2007. Retaining desirable properties in discretising a travel-time model, Transportation Research Part B: Methodological 41(5): 540-553. http://dx.doi.org/10.1016/j.trb.2006.09.003

Carey, M.; Ge, Y. E.; McCartney, M. 2003. A whole-link traveltime model with desirable properties, Transportation Science 37(1): 83-96.

http://dx.doi.org/10.1287/trsc.37.1.83.12819

Carey, M.; Humphreys, P.; McHugh, M.; McIvor, R. 2014. Extending travel-time based models for dynamic network loading and assignment, to achieve adherence to firstin-first-out and link capacities, Transportation Research Part B: Methodological 65: 90-104. http://dx.doi.org/10.1016/j.trb.2014.04.002

Chabini, I. 2001. Analytical dynamic network loading problem: formulation, solution algorithms, and computer implementations, Transportation Research Record 1771: 191200. http://dx.doi.org/10.3141/1771-24

Daganzo, C. F. 1995. The cell transmission model, part II: network traffic, Transportation Research Part B: Methodological 29(2): 79-93. http://dx.doi.org/10.1016/0191-2615(94)00022-R

Daganzo, C. F. 1994. The cell transmission model: a dynamic representation of highway traffic consistent with the hydrodynamic theory, Transportation Research Part B: Methodological 28(4): 269-287. http://dx.doi.org/10.1016/0191-2615(94)90002-7

Drissi-Kaïtouni, O.; Hameda-Benchekroun, A. 1992. A dynamic traffic assignment model and a solution algorithm, Transportation Science 26(2): 119-128. http://dx.doi.org/10.1287/trsc.26.2.119

Friesz, T. L.; Bernstein, D.; Smith, T. E.; Tobin, R. L.; Wie, B. W. 1993. A variational inequality formulation of the dynamic network user equilibrium problem, Operations Research 41(1): 179-191. http://dx.doi.org/10.1287/opre.41.1.179

Friesz, T. L.; Luque, J.; Tobin, R. L.; Wie, B.-W. 1989. Dynamic network traffic assignment considered as a continuous time optimal control problem. Operations Research 37(6): 893901. http://dx.doi.org/10.1287/opre.37.6.893

Han, K.; Friesz, T. L.; Yao, T. 2013a. Existence of simultaneous route and departure choice dynamic user equilibrium, Transportation Research Part B: Methodological 53: 17-30. http://dx.doi.org/10.1016/j.trb.2013.01.009 
Han, K.; Friesz, T. L.; Yao, T. 2013b. A partial differential equation formulation of Vickrey's bottleneck model, part I: Methodology and theoretical analysis, Transportation Research Part B: Methodological 49: 55-74. http://dx.doi.org/10.1016/j.trb.2012.10.003

Han, K.; Friesz, T. L.; Yao, T. 2013c. A partial differential equation formulation of Vickrey's bottleneck model, part II: Numerical analysis and computation, Transportation Research Part B: Methodological 49: 75-93. http://dx.doi.org/10.1016/j.trb.2012.10.004

Kuwahara, M.; Akamatsu, T. 1997. Decomposition of the reactive dynamic assignments with queues for a many-to-many origin-destination pattern, Transportation Research Part B: Methodological 31(1): 1-10. http://dx.doi.org/10.1016/S0191-2615(96)00020-3

Lighthill, M. J.; Whitham, G. B. 1955. On kinematic waves. II. A theory of traffic flow on long crowded roads, Proceedings of the Royal Society A: Mathematical, Physical \& Engineering Sciences 229: 317-345.

http://dx.doi.org/10.1098/rspa.1955.0089

Logghe, S.; Immers, L. H. 2008. Multi-class kinematic wave theory of traffic flow, Transportation Research Part B: Methodological 42(6): 523-541. http://dx.doi.org/10.1016/j.trb.2007.11.001

Merchant, D. K.; Nemhauser, G. L. 1978. A model and an algorithm for the dynamic traffic assignment problems, Transportation Science 12(3): 183-199. http://dx.doi.org/10.1287/trsc.12.3.183

Newell, G. F. 1993a. A simplified theory of kinematic waves in highway traffic, part I: general theory, Transportation Research Part B: Methodological 27(4): 281-287. http://dx.doi.org/10.1016/0191-2615(93)90038-C

Newell, G. F. 1993b. A simplified theory of kinematic waves in highway traffic, part II: queueing at freeway bottlenecks, Transportation Research Part B: Methodological 27(4): 289-303. http://dx.doi.org/10.1016/0191-2615(93)90039-D

Newell, G. F. 1993c. A simplified theory of kinematic waves in highway traffic, part III: multi-destination flows, Transportation Research Part B: Methodological 27(4): 305-313. http://dx.doi.org/10.1016/0191-2615(93)90040-H

Peeta, S.; Ziliaskopoulos, A. K. 2001. Foundations of dynamic traffic assignment: the past, the present and the future, Networks and Spatial Economics 1(3-4): 233-265. http://dx.doi.org/10.1023/A:1012827724856

Smith, M. J. 1984. The existence of a time-dependent equilibrium distribution of arrivals at a single bottleneck, Transportation Science 18(4): 385-394. http://dx.doi.org/10.1287/trsc.18.4.385

Smits, E.-S.; Bliemer, M.; Van Arem, B. 2011. Dynamic network loading of multiple user-classes with the link transmission model, Proceedings of the 2nd International Conference on Models and Technologies for Intelligent Transportation Systems, 22-24 June 2011, Leuven, Belgium, 1-4.

Vickrey, W. S. 1969. Congestion theory and transport investment, The American Economic Review 59(2): 251-260.

Wu, J. H.; Chen, Y.; Florian, M. 1998. The continuous dynamic network loading problem: a mathematical formulation and solution method, Transportation Research Part B: Methodological 32(3): 173-187. http://dx.doi.org/10.1016/S0191-2615(97)00023-4

Yperman, I. 2007. The Link Transmission Model for Dynamic Network Loading: PhD Dissertation. Catholic University of Leuven, Belgium. 178 p. Available from Internet: http:// www.kuleuven.be/traffic/dwn/P2007A
Zhang, H. M.; Nie, Y.; Qian, Z. 2013. Modelling network flow with and without link interactions: the cases of point queue, spatial queue and cell transmission model, Transportmetrica B: Transport Dynamics 1(1): 33-51. http://dx.doi.org/10.1080/21680566.2013.785921 